OPEN ACCESS

Edited by:

Xiuming Jin,

Zhejiang University, China

Reviewed by:

Baihua Chen,

Central South University, China

Gui-shuang Ying,

University of Pennsylvania,

United States

${ }^{*}$ Correspondence:

Peng Shen

shen-peng@foxmail.com

Hongbo Lin

lin673160@163.com

Wei Chen

chenweimd@wmu.edu.cn

†These authors have contributed equally to the study

Specialty section:

This article was submitted to Ophthalmology,

a section of the journal

Frontiers in Medicine

Received: 10 December 2021

Accepted: 10 January 2022

Published: 04 February 2022

Citation:

Zheng Q, Li S, Wen F, Lin Z, Feng K

Sun $Y$, Bao J, Weng $H$, Shen P, Lin H and Chen W (2022) The Association

Between Sleep Disorders and

Incidence of Dry Eye Disease in

Ningbo: Data From an Integrated

Health Care Network.

Front. Med. 9:832851.

doi: 10.3389/fmed.2022.832851

\section{The Association Between Sleep Disorders and Incidence of Dry Eye Disease in Ningbo: Data From an Integrated Health Care Network}

\author{
Qinxiang Zheng ${ }^{1,2+}$, Saiqing $\mathrm{Li}^{2 \dagger}$, Feng Wen ${ }^{1+}$, Zhong $\mathrm{Lin}^{2}$, Kemi Feng ${ }^{2}$, Yexiang Sun ${ }^{3}$, \\ Jie Bao ${ }^{2}$, Hongfei Weng ${ }^{1}$, Peng Shen ${ }^{3 *}$, Hongbo Lin ${ }^{3 *}$ and Wei Chen ${ }^{1,2 *}$ \\ ${ }^{1}$ The Affiliated Ningbo Eye Hospital of Wenzhou Medical University, Ningbo, China, ${ }^{2}$ Eye Hospital and School of \\ Ophthalmology and Optometry, Wenzhou Medical University, Wenzhou, China, ${ }^{3}$ Department of Chronic Diseases and Health \\ Promotion, Yinzhou District Center for Disease Control and Prevention, Ningbo, China
}

Purpose: To investigate the association between sleep disorders and dry eye disease (DED) in Ningbo, China.

Methods: Our data came from the Yinzhou Health Information System (HIS), including 257932 patients and was based on a 1:1 matching method (sleep disorder patients vs. patients without sleep disorders) during 2013-2020. Sleep disorders and DED were identified using ICD-10 codes. Cox proportional hazards regression was used to identify the association between sleep disorders and DED.

Results: The eight-year incidence of DED was significantly higher in participants with diagnosis of sleep disorders (sleep disorders: 50.66\%, no sleep disorders: $16.48 \%$, $P<0.01)$. Sleep disorders were positively associated with the diagnosis of DED (HR: 3.06, 95\% Cl: 2.99-3.13, $P<0.01$ ), when sex, age, hypertension, diabetes and other systemic diseases were adjusted. In the sleep disorders patients, advancing age, female sex, and presence of coexisting disease (hypertension, diabetes, hyperlipidemia, thyroid disease, depression, heart disease, and arthritis) were significantly associated with the development of DED by the multivariate cox regression analysis (all $P<0.05$ ). In addition, there was a significantly positive association between estazolam and the incidence of DED in both sleep disorder and non-sleep disorder groups (all $P<0.05$ ).

Conclusions : Sleep disrder was associated with a three-time increased risk of DED. This association can be helpful in effective management of both sleep disorders and DED.

Keywords: sleep disorders, dry eye disease, risk factor, sleep medicine, coexisting disease

\section{INTRODUCTION}

Sleep disorder, defined as an abnormality in sleep quality or quantity, has become a major social health concern throughout the world (1-3). Sleep quality and duration of sleep are important for physical and mental health, and sleep dysfunction damages autonomic nerve and endocrine function, leading to extensive changes in many aspects of the body system (3). Numerous evidence show that sleep disorders increase the risk of obesity, hypertension, diabetes, dementia, and cardiovascular disease (4-9). 
Dry eye disease (DED) is a multifactorial disease of the ocular surface characterized by a loss of tear film homeostasis, causing tear film instability and hyperosmolarity, as well as ocular surface inflammation and damage, and neurosensory abnormalities play etiological roles (10). The incidence of DED was estimated to range from 5 to $50 \%$ worldwide (11). DED causes a variety of ocular surface discomfort (such as pain, foreign body sensation, irritation, and photophobia) and visual dysfunction, which not only impedes daily activities, but also exerts psychological impacts (12-14).

Previous studies have found that aging, female sex, systemic diseases (hypertension, diabetes, thyroid disease, anxiety, depression, and so on) are associated with DED development $(11,12,15,16)$. The association between DED and sleep disorders has recently attracted attention $(11,17,18)$. It was found that DED patients sleep poorly, and patients with sleep disorders are more likely to have DED $(17,19-28)$. Some studies were conducted in community populations or cohort populations by collecting questionnaires on sleep and dry eye symptoms $(19,20,26,27)$, however these studies may have drawbacks of selection bias and recall bias. There are also studies suggesting that sedative antidepressants would promote dry eye symptoms and signs $(17,29-31)$. In the current study, we would like to compare the incidence of DED that developed in participants with or without sleep disorders, using the integrated health care network system data, and evaluate whether sleep disorders was a risk factor for DED.

\section{MATERIALS AND METHODS}

\section{Study Population}

Yinzhou District, located in the Yangtze River Delta, is one of the core areas of Ningbo. According to the statistical yearbook of Yinzhou District, by the end of 2018, there were 1.34 million permanent residents in Yinzhou District. Data on hospital visits were obtained from the Yinzhou Health Information System (YHIS), which seamlessly links the health information archives, chronic disease registration system, resident death registration system and hospital outpatient system of permanent residents in Yinzhou District, and can provide real-time treatment information of patients in 2 general hospitals, 3 specialized hospitals, 24 community health service centers and 248 community service stations in the whole district. The health information of each resident living in Yinzhou is integrated into YHIS. Detailed information about this system has been described in the previous research (32).

Medical information was classified according to the ICD10 (International Classification of Diseases, 10th Revision). In this study, we first excluded all patients who were deceased, we selected hospital visits for sleep disorders (ICD-10: G47) and DED (ICD-10: H04.103, H11.104, H16.202 and H18.803) during 2013-2020. We excluded patients diagnosed with DED before sleep disorders. Finally, we identified 128,966 patients with sleep disorders between 2013 and 2020. To design the matched cohort study using the sleep disorder patients as the case group, we established the control group by propensity score matching by adjusting for same sex, same age range and same date of diagnosis for sleep disorders. We used a 1:1 matching method to select the control group $(128,966$ patients) who were not diagnosed with sleep disorders. Finally, the data used in this study included 257,932 patients from 2013 to 2020 .

\section{Variables}

The outcome variable of this study is whether DED will occur after patients were diagnosed with sleep disorders. If

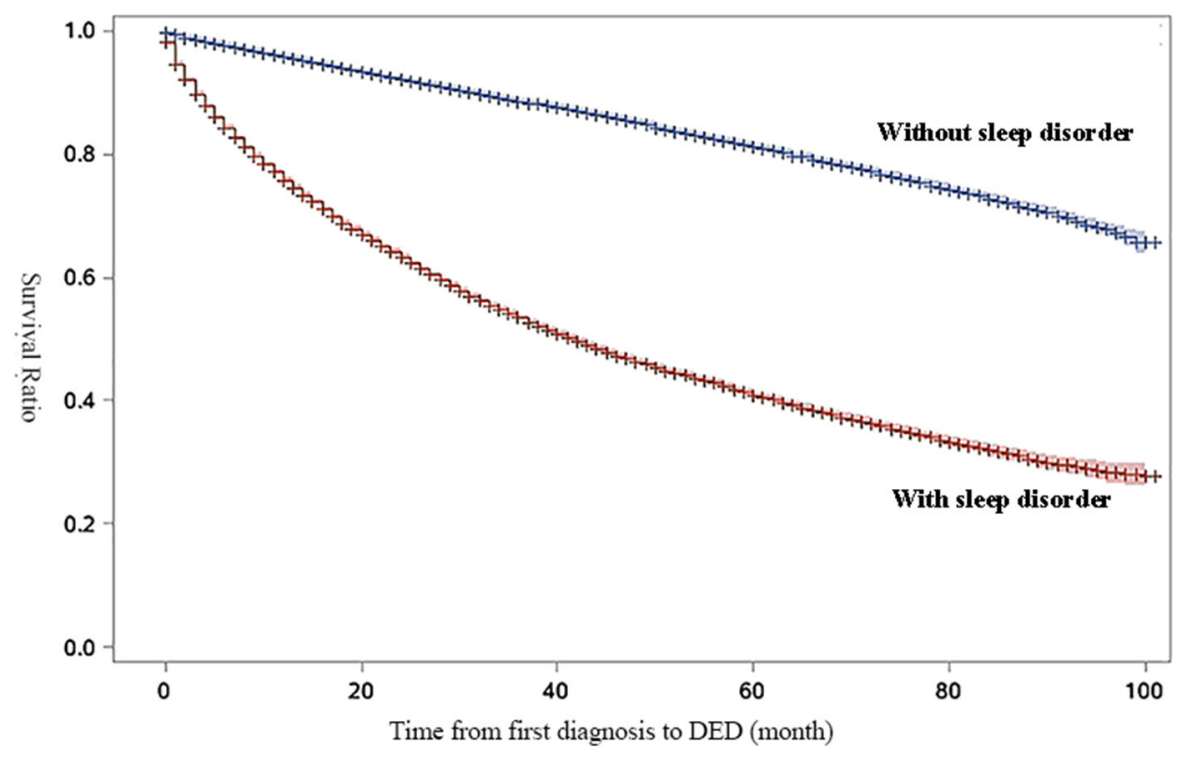

FIGURE 1 | Survival curve for survival-ratio of dry eye disease (DED). The results of log-rank test for time to DED by diagnosis of sleep disorders were statistically significant (HR: 3.81, 95\%Cl:3.75-3.87, $P<0.01)$. 
diagnosis of DED (ICD-10: H04.103, H11.104, H16.202, and H18.803), it was defined as DED diagnosis, and classified the patient as the DED diagnosis group. In this study, sleep disorders were defined as organic sleep disorders (ICD-10: G47), including insomnia, hypersomnia, narcolepsy/cataplexy, sleep apnea, disorders of the sleep-wake schedule and other sleep disorders. In the study, we included the following independent variables: gender, age, medical history (hypertension, diabetes, thyroid disease, anxiety, depression, heart disease, and arthritis), and medication history of sleep disorders. The ages were classified at 10-year intervals: under 29 years, 30-39 years, 4049 years, 50-59 years, 60-69 years, over 70 years. At present, the clinical drugs for insomnia mainly include benzodiazepine receptor agonists (BZRAs), melatonin receptor agonists, orexin receptor antagonists, antidepressants and etc. $(33,34)$. We studied the using condition of sleep medicine in patients with sleep disorders, excluding the drugs mentioned in the guide but not found in the YHIS. Finally, we mainly included BZRAs (estazolam, diazepam, alprazolam, lorazepam), antidepressants (trazodone, mirtazapine, doxepin), and other sedatives (gabapentin, olanzapine).

\section{Statistical Analysis}

In order to study the relationship between DED and sleep disorders, we first determined the frequency and percentage of people with DED diagnosis. Next, we performed a log rank test and generated a survival curve to compare the diagnostic rate of DED between patients with and without sleep disorders. Finally, we performed survival analysis using the univariate cox regression analysis, and then variables with $P<0.20$ in univariate regression analysis were included in multivariate cox regression analysis to determine the association between sleep disorders and DED, and studied the association of other independent variables with DED. All statistical analyses were performed using SAS statistical software version 9.2 (SAS company, Cary, North Carolina, USA). A $P<0.05$ was considered to be statistically significant.

\section{RESULTS}

Using the 1:1 propensity score matching method (patients with sleep disorder vs. patients without sleep disorders), we analyzed 257,932 patients with or without sleep disorders between 2013 and 2020. Supplemental Table 1 shows the distribution of the study population for each variable and the 8-year incidence of DED among different variable subgroups. The 8-year incidence of DED was 50.66 and $16.48 \%$ in participants with and without sleep disorders, respectively. About $33.57 \%$ of patients in our study were diagnosed with DED over 8 years, and incidence

TABLE 1 | Results of survival analysis using Cox regression analysis for association between sleep disorders and dry eye disease (DED) in the study population ( $n=$ 257,932).

\begin{tabular}{|c|c|c|c|c|c|c|}
\hline \multirow[t]{2}{*}{ Variables } & \multicolumn{2}{|c|}{ Model 1} & \multicolumn{2}{|c|}{ Model 2} & \multicolumn{2}{|c|}{ Model 3} \\
\hline & HR (95\%Cl) & $\boldsymbol{P}$ & HR $(95 \% \mathrm{Cl})$ & $\boldsymbol{P}$ & HR (95\%Cl) & $P$ \\
\hline Sleep disorder & $3.81(3.75,3.87)$ & $<0.01$ & $3.28(3.23,3.34)$ & $<0.01$ & $3.06(2.99,3.13)$ & $<0.01$ \\
\hline \multicolumn{7}{|l|}{ Sex } \\
\hline Male & $1.00(-)$ & - & - & - & $1.00(-)$ & - \\
\hline Female & $1.20(1.17,1.23)$ & $<0.01$ & - & - & $1.25(1.22,1.29)$ & $<0.01$ \\
\hline \multicolumn{7}{|l|}{ Age (years) } \\
\hline$<30$ & $1.0(-)$ & - & - & - & $1.0(-)$ & - \\
\hline 30-39 & $1.86(1.71,2.04)$ & $<0.01$ & - & - & $1.94(1.77,2.12)$ & $<0.01$ \\
\hline $40-49$ & $2.00(1.88,2.13)$ & $<0.01$ & - & - & $2.04(1.92,2.18)$ & $<0.01$ \\
\hline $50-59$ & $1.71(1.62,1.82)$ & $<0.01$ & - & - & $1.73(1.63,1.84)$ & $<0.01$ \\
\hline $60-69$ & $1.44(1.36,1.53)$ & $<0.01$ & - & - & $1.46(1.38,1.55)$ & $<0.01$ \\
\hline$\geq 70$ & $1.18(1.11,1.26)$ & $<0.01$ & - & - & $1.18(1.11,1.26)$ & $<0.01$ \\
\hline \multicolumn{7}{|l|}{ Medical history } \\
\hline Hypertension & $1.12(1.17,1.23)$ & $<0.01$ & $1.04(0.98,1.10)$ & 0.21 & $1.02(0.96,1.08)$ & 0.51 \\
\hline Diabetes & $1.07(0.98,1.16)$ & 0.12 & $0.96(0.88,1.04)$ & 0.33 & $0.95(0.87,1.03)$ & 0.19 \\
\hline Hyperlipidemia & $1.22(1.12,1.34)$ & $<0.01$ & $1.10(1.01,1.20)$ & 0.04 & $1.09(0.99,1.19)$ & 0.07 \\
\hline Thyroid disease & $0.91(0.70,1.17)$ & 0.44 & $0.85(0.66,1.10)$ & 0.21 & - & - \\
\hline Anxiety & $1.13(0.76,1.69)$ & 0.54 & $0.98(0.65,1.46)$ & 0.90 & - & - \\
\hline Depression & $1.19(0.91,1.56)$ & 0.19 & $1.15(0.88,1.50)$ & 0.31 & $1.14(0.87,1.50)$ & 0.33 \\
\hline Heart disease & $1.38(1.22,1.56)$ & $<0.01$ & $1.20(1.07,1.36)$ & $<0.01$ & $1.18(1.05,1.34)$ & $<0.01$ \\
\hline Arthritis & $1.39(1.01,1.93)$ & 0.04 & $1.23(0.89,1.70)$ & 0.21 & $1.23(0.89,1.70)$ & 0.21 \\
\hline
\end{tabular}

HR, Hazard ratio; Cl, Confidence interval.

Model 1: Univariate cox regression analysis, and no variables were adjusted.

Model 2: Sex and age were adjusted.

Model 3: Variables with $P<0.20$ in univariate cox regression analysis were included for multivariate cox regression analysis. 

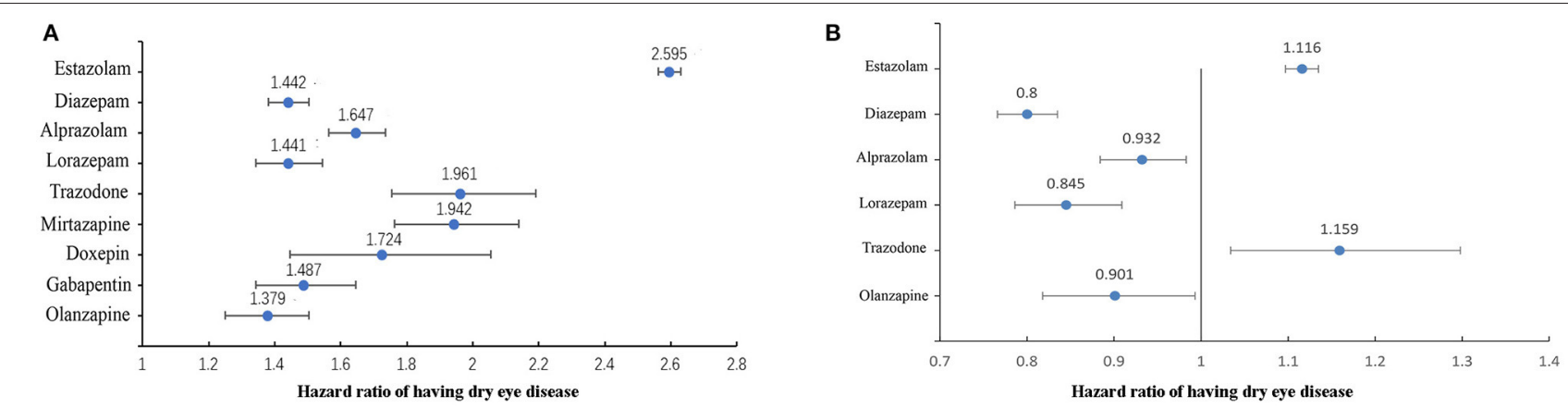

FIGURE 2 | The association between sleep medicine and DED in general population ( $n=257,932)$. (A) Results of univariate cox regression analysis, all drugs in the figure were statistically significant $(P<0.01)$. (B) Results of multivariate cox regression analysis, all drugs in the figure were statistically significant $(P<0.05)$.

TABLE 2 | The distribution of the sleep disorders population $(n=128,966)$ for each sleep medicine.

\begin{tabular}{|c|c|c|c|c|c|}
\hline \multirow[t]{2}{*}{ Medicine } & \multicolumn{2}{|c|}{ Total } & \multicolumn{2}{|c|}{ DED } & \multirow[t]{2}{*}{ P-value } \\
\hline & $N$ & $\%$ & $N$ & $\%$ & \\
\hline Estazolam & & & & & $<0.01$ \\
\hline Yes & 81,539 & 63.23 & 44,116 & 54.10 & \\
\hline No & 47,427 & 36.77 & 21,222 & 44.75 & \\
\hline Diazepam & & & & & $<0.01$ \\
\hline Yes & 3,626 & 2.81 & 2,188 & 60.34 & \\
\hline No & 125,340 & 97.19 & 63,150 & 50.38 & \\
\hline Alprazolam & & & & & $<0.01$ \\
\hline Yes & 2,346 & 1.82 & 1,429 & 60.91 & \\
\hline No & 126,620 & 98.18 & 63,909 & 50.47 & \\
\hline Lorazepam & & & & & $<0.01$ \\
\hline Yes & 1,288 & 1.00 & 775 & 60.17 & \\
\hline No & 127,678 & 99.00 & 64,563 & 50.57 & \\
\hline Trazodone & & & & & $<0.01$ \\
\hline Yes & 505 & 0.39 & 311 & 61.58 & \\
\hline No & 128,461 & 99.61 & 65,027 & 50.62 & \\
\hline Mirtazapine & & & & & $<0.01$ \\
\hline Yes & 616 & 0.48 & 402 & 65.26 & \\
\hline No & 128,350 & 99.52 & 64,936 & 50.59 & \\
\hline Doxepin & & & & & $<0.01$ \\
\hline Yes & 174 & 0.13 & 124 & 71.26 & \\
\hline No & 128,792 & 99.87 & 65,214 & 50.64 & \\
\hline Gabapentin & & & & & $<0.01$ \\
\hline Yes & 502 & 0.39 & 293 & 58.37 & \\
\hline No & 128,464 & 99.61 & 65,045 & 50.63 & \\
\hline Olanzapine & & & & & $<0.01$ \\
\hline Yes & 754 & 0.58 & 429 & 56.90 & \\
\hline No & 128,212 & 99.42 & 64,909 & 50.63 & \\
\hline
\end{tabular}

The proportion of DED for different sleep medicine in sleep disorders population $(n=128,966)$.

DED, Dry eye disease.

${ }^{*}$ Chi square test was used to compare the incidence of DED with the use of individual drug. was higher in patients with sleep disorders (with sleep disorders: $50.66 \%$, without sleep disorders: $16.48 \%)$.

Figure 1 shows the survival curve to compare the survival rate of DED in patients with sleep disorders and without sleep disorders during the study period. In the survival curve, patients with sleep disorders were more likely to be diagnosed with DED than patients without sleep disorders $(P<0.05$ for log rank test).

Table 1 shows the results of survival analysis using the Cox proportional hazard model to determine the association between DED and sleep disorders after adjusting other independent variables. Univariate regression analysis showed that sleep disorders, age, gender and coexisting systemic diseases (hypertension, hyperlipidemia, heart disease, and arthritis) were significantly associated with the development of DED (all $P<$ $0.05)$. Then we included variables with $P<0.20$ from the model 1 for multivariate cox regression analysis. By the multivariate regression analysis, sleep disorders were positively associated with the diagnosis of DED (HR: 3.06, 95\% CI: 2.99-3.13, $P<$ 0.01 ), with a three-time increased risk. Among the independent variables, the risk of diagnosing DED was higher in women than in men (HR: 1.25, 95\% CI: 1. 22-1.29, $P<0.01$ ). Compared with young people who were $<30$ years, increased age was associated with DED diagnosis $(P<0.01)$, and the risk was the highest in the population aged $40-49$ years (HR: $2.04,95 \%$ CI: $1.92-2.18, P$ $<0.01)$. In addition, only heart disease was positively associated with the diagnosis of DED $(P<0.01)$. At the same time, other coexisting systematic diseases did not have significant association with the diagnosis of DED $(P>0.05)$.

Next, we analyzed the effect of sleep drugs on DED in the general population. Figure 2A shows the results of univariate cox regression analysis, all sleep medicine increase the risk of DED (all $P<0.01$ ). The sleep medicine with statistical significance $(P<0.05)$ in multivariate cox regression analysis are shown in Figure 2B. It can be seen that estazolam and trazodone were positively associated with the DED.

Additionally, we analyzed the using condition of sleep medicine and its association with DED in sleep disorders 


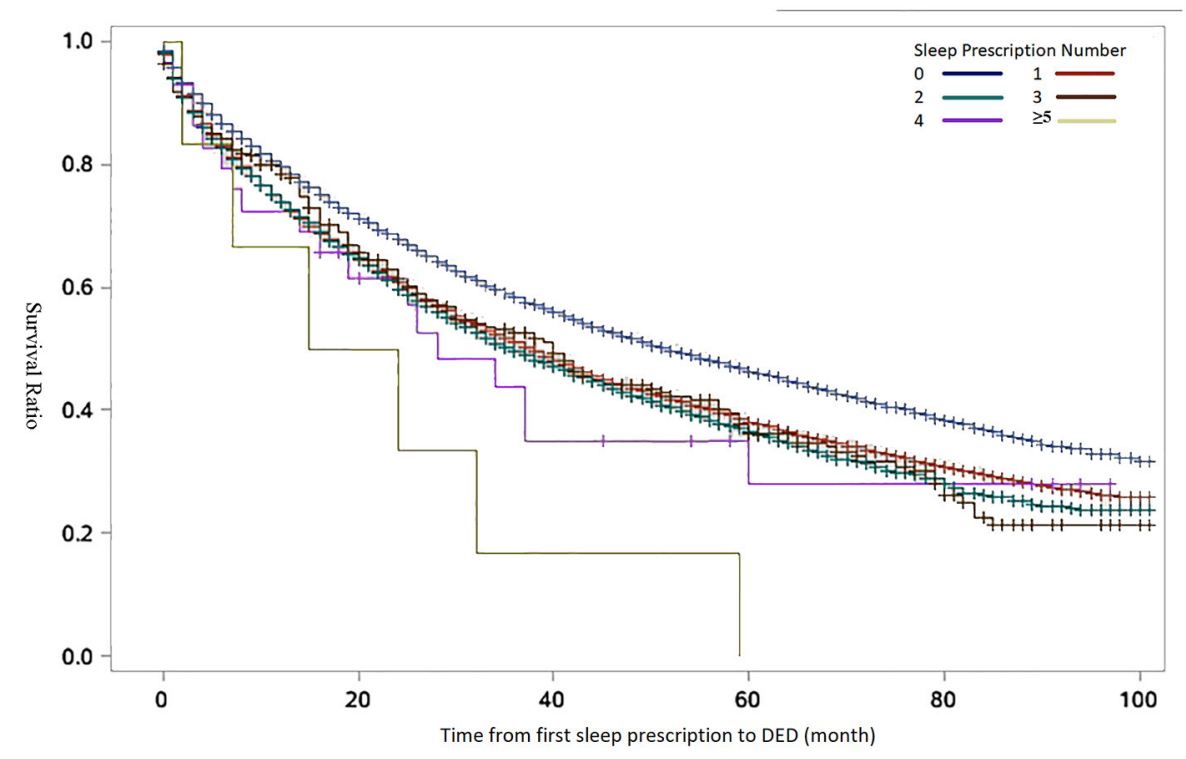

FIGURE 3 | Kaplan-Meier curves for survival-ratio of dry eye disease (DED) over time by the number of sleep prescriptions. The results of log-rank test for time to DED by sleep prescriptions number were statistically significant $(P<0.01)$. The number of sleep prescription refers to the cumulative number of sleep drugs used by the patient.

patients. Table 2 shows the distribution of the sleep disorders population for each sleep medicine with the Chi square test. The number of cases in Table 2 refers to the person who uses this sleep drug, regardless of whether they use other type sleep drugs or not. Among the sleep disorders, the frequency of estazolam was $63.23 \%$, which was most common in the patients. Compared with patients not using sleep medicine, the incidence of DED significantly increased in the sleep medicine using group (all $P<$ 0.01 ). Figure 3 shows that the incidence of DED over time raised with the increasing of the sleep prescription number used in the sleep disorders.

Similarly, we performed Cox proportional hazard model in the sleep disorder group to determine the relationship between the independent variable and DED. The results of regression analysis are presented in the Table 3 . In the model 3 (variables with $P<0.20$ in univariate cox analysis were included for multivariate cox analysis), the variables of female and 30-39 years significantly increased the DED diagnosis $(P<0.01)$ in the sleep disorders patients. However, the results comparing anxiety with DED were not statistically significant $(P=0.08)$. As is shown in Table 3, the estazolam and trazodone were positively associated with the DED $(P<0.05)$. Contrarily, diazepam and lorazepam showed negative correlation with DED $(P<0.05)$.

In the same way, we analyzed the relationship between the independent variable and DED in the patients without sleep disorder by performing Cox proportional hazard model. The results of regression analysis are presented in the Table 4. We can see the variables of female and 40-49 years significantly increased the DED diagnosis $(\mathrm{P}<0.01)$ when using multivariate cox analysis. Among the coexisting systemic diseases, only heart disease was significantly correlated with the incidence of DED $(P=0.01)$. Table 4 showed the estazolam, diazepam and gabapentin were all positively associated with the DED $(P<0.05)$.

\section{DISCUSSION}

In the current study, we found that sleep disorder is a risk factor of DED, with a three-time increased risk to the subjects without sleep disorders. Besides, the use of estazolam increased the risk of DED regardless of the presence of sleep disorder.

In our study, the 8-year incidence of DED in the study population was $33.57 \%$. Many previous population-based studies have indicated a correlation between sleep disorders and DED $(19,20,24-27,35)$. Han et.al reported that sleep disorders were related to dry eye incidence rate (HR:1.32), and sleep had a greater impact on DED in male and young patients (36). In a nationally population-based survey in Korea, people with sleep duration shorter than $5 \mathrm{~h}$ were found to be $20 \%$ more likely to suffer from DED, compared to those with more than $6 \mathrm{~h}$ of sleep (35).Hanyuda et al. also showed that sleep deprivation and poor sleep quality were significantly related to DED in a Japanese population (26). Additionally, two large studies conducted in Hangzhou and Singapore also revealed that poor sleep quality and short sleep time were significantly correlated with DED (19, 20). Our results are similar with the above studies, compared with patients without sleep disorders, patients with sleep disorders have a higher risk of DED (HR:3.06).

The association between sleep disorders and DED are based on physiological findings. Sleep disorders are often related to autonomic nerve dysfunction, which could affect 
TABLE 3 | Results of survival analysis using Cox proportional hazard model for association between independent variables and dry eye disease (DED) in the sleep disorder patients $(n=128,966)$.

\begin{tabular}{|c|c|c|c|c|c|c|}
\hline \multirow[t]{2}{*}{ Variables } & \multicolumn{2}{|c|}{ Model 1} & \multicolumn{2}{|c|}{ Model 2} & \multicolumn{2}{|c|}{ Model 3} \\
\hline & HR $(95 \% \mathrm{Cl})$ & $P$ & HR $(95 \% \mathrm{Cl})$ & $P$ & HR $(95 \% \mathrm{Cl})$ & $P$ \\
\hline \multicolumn{7}{|l|}{ Sex } \\
\hline Male & $1.00(-)$ & - & - & - & $1.00(-)$ & - \\
\hline Female & $1.14(1.12,1.16)$ & $<0.01$ & - & - & $1.15(1.13,1.17)$ & $<0.01$ \\
\hline \multicolumn{7}{|l|}{ Age (years) } \\
\hline$<30$ & $1.00(-)$ & - & - & - & $1.00(-)$ & - \\
\hline 30-39 & $1.84(1.74,1.95)$ & $<0.01$ & - & - & $1.70(1.60,1.81)$ & $<0.01$ \\
\hline $40-49$ & $1.80(1.70,1.90)$ & $<0.01$ & - & - & $1.67(1.57,1.77)$ & $<0.01$ \\
\hline $50-59$ & $1.51(1.42,1.60)$ & $<0.01$ & - & - & $1.42(1.33,1.50)$ & $<0.01$ \\
\hline $60-69$ & $1.32(1.25,1.40)$ & $<0.01$ & - & - & $1.28(1.21,1.36)$ & $<0.01$ \\
\hline$\geq 70$ & $1.23(1.15,1.31)$ & $<0.01$ & - & - & $1.20(1.13,1.28)$ & $<0.01$ \\
\hline \multicolumn{7}{|l|}{ Medical history } \\
\hline Hypertension & $1.10(1.08,1.12)$ & $<0.01$ & $0.94(0.93,0.96)$ & $<0.01$ & $0.87(0.86,0.89)$ & $<0.01$ \\
\hline Diabetes & $1.07(1.05,1.09)$ & $<0.01$ & $0.99(0.97,1.01)$ & 0.32 & $0.97(0.95,0.99)$ & $<0.01$ \\
\hline Hyperlipidemia & $1.22(1.20,1.24)$ & $<0.01$ & $1.13(1.11,1.15)$ & $<0.01$ & $1.11(1.09,1.13)$ & $<0.01$ \\
\hline Thyroid disease & $1.10(1.04,1.17)$ & $<0.01$ & $1.12(1.05,1.19)$ & $<0.01$ & $1.10(1.04,1.17)$ & $<0.01$ \\
\hline Anxiety & $1.09(1.08,1.11)$ & $<0.01$ & $1.04(1.02,1.06)$ & $<0.01$ & $0.98(0.96,1.00)$ & 0.08 \\
\hline Depression & $1.18(1.15,1.20)$ & $<0.01$ & $1.15(1.13,1.18)$ & $<0.01$ & $1.13(1.10,1.16)$ & $<0.01$ \\
\hline Heart disease & $1.27(1.25,1.29)$ & $<0.01$ & $1.16(1.15,1.18)$ & $<0.01$ & $1.15(1.13,1.17)$ & $<0.01$ \\
\hline Arthritis & $1.18(1.14,1.23)$ & $<0.01$ & $1.15(1.10,1.20)$ & $<0.01$ & $1.12(1.08,1.17)$ & $<0.01$ \\
\hline \multicolumn{7}{|c|}{ Sleep medicines } \\
\hline Estazolam & $1.21(1.19,1.23)$ & $<0.01$ & $1.12(1.10,1.20)$ & $<0.01$ & $1.12(1.10,1.14)$ & $<0.01$ \\
\hline Diazepam & $0.87(0.83,0.90)$ & $<0.01$ & $0.84(0.81,0.88)$ & $<0.01$ & $0.80(0.77,0.83)$ & $<0.01$ \\
\hline Alprazolam & $1.01(0.96,1.06)$ & 0.80 & $0.98(0.93,1.03)$ & 0.36 & - & - \\
\hline Lorazepam & $0.94(0.87,1.00)$ & 0.06 & $0.92(0.85,0.98)$ & $<0.01$ & $0.83(0.78,0.90)$ & $<0.01$ \\
\hline Trazodone & $1.26(1.12,1.40)$ & $<0.01$ & $1.25(1.11,1.40)$ & $<0.01$ & $1.15(1.03,1.29)$ & 0.02 \\
\hline Mirtazapine & $1.20(1.09,1.33)$ & $<0.01$ & $1.18(1.06,1.30)$ & $<0.01$ & $1.09(0.99,1.21)$ & 0.09 \\
\hline Doxepin & $1.04(0.87,1.24)$ & 0.65 & $0.96(0.80,1.15)$ & 0.66 & - & - \\
\hline Gabapentin & $1.12(1.05,1.30)$ & 0.27 & $1.09(0.97,1.22)$ & 0.12 & - & - \\
\hline Olanzapine & $0.98(0.89,1.08)$ & 0.72 & $0.96(0.88,1.06)$ & 0.44 & - & - \\
\hline
\end{tabular}

HR, Hazard ratio; Cl, Confidence interval.

Model 1: Univariate cox regression analysis, and no variables were adjusted.

Model 2: Sex and age adjusted.

Model 3: Variables with $P<0.20$ in univariate cox analysis were included for multivariate cox analysis.

the parasympathetic fibers in the lacrimal gland, resulting in reduced tear secretion $(37,38)$. Sleep deprivation may also inhibit peroxisome proliferator-activated receptor alpha $(P P A R \alpha)$ signaling which increase oxidative stress leading to DED (39). In addition, the activation of the hypothalamic-pituitary-adrenal axis during sleep may lead to a relatively dehydrated state, thereby reducing tear secretion $(40,41)$.

Our results also found that use of sleep medications in the sleep disorders was significantly associated with DED. Kawashima et al. have demonstrated that sedative antidepressants could significantly decreased tear break-up time of DED patients (29). Antidepressants could promote dry eye symptoms through anticholinergic side effects (inhibition of cholinergic nerve fibers in meibomian and lacrimal glands) or other potential pharmacological mechanisms to reduce tear secretion $(17,30)$. Using the multivariate cox regression analysis, we found that there was a significantly positive association between estazolam and the incidence of DED in both sleep disorder and non-sleep disorder participants. Estazolam, a kind of benzodiazepine drugs (BZDs), can non selectively activate different $\gamma$ subunits on $\gamma$-aminobutyric acid receptor $\mathrm{A}$ and has pharmacological effects of sedation, hypnosis, antianxiety, muscle relaxation and anti-convulsion (42). Estazolam has side effects of anticholinergic activity (43), which reduces the secretion of tears, resulting in DED. In the present study, the ratio of estazolam use amounts to $63.23 \%$ in the subjects with sleep disorders, while the ratio of other sleep medicine were below 3\%, which may limit the power of detection small effect. Therefore, the association between other sleep medicines and dry eye needs to be evaluated in a larger study with sufficient sleep medication users. 
TABLE 4 | Results of survival analysis using Cox proportional hazard model for association between independent variables and dry eye disease (DED) in the patients without sleep disorder ( $n=128,966)$.

\begin{tabular}{|c|c|c|c|c|c|c|}
\hline \multirow[t]{2}{*}{ Variables } & \multicolumn{2}{|c|}{ Model 1} & \multicolumn{2}{|c|}{ Model 2} & \multicolumn{2}{|c|}{ Model 3} \\
\hline & HR $(95 \% \mathrm{Cl})$ & $P$ & HR $(95 \% \mathrm{Cl})$ & $P$ & HR $(95 \% \mathrm{Cl})$ & $P$ \\
\hline \multicolumn{7}{|l|}{ Sex } \\
\hline Male & $1.00(-)$ & - & - & - & $1.00(-)$ & - \\
\hline Female & $1.20(1.17,1.23)$ & $<0.01$ & - & - & $1.25(1.22,1.29)$ & $<0.01$ \\
\hline \multicolumn{7}{|l|}{ Age (years) } \\
\hline$<30$ & $1.00(-)$ & - & - & - & $1.00(-)$ & - \\
\hline 30-39 & $1.86(1.71,2.04)$ & $<0.01$ & - & - & $1.92(1.75,2.10)$ & $<0.01$ \\
\hline $40-49$ & $2.00(1.88,2,13)$ & $<0.01$ & - & - & $2.03(1.90,2.16)$ & $<0.01$ \\
\hline $50-59$ & $1.71(1.62,1.82)$ & $<0.01$ & - & - & $1.72(1.62,1.83)$ & $<0.01$ \\
\hline $60-69$ & $1.44(1.36,1.53)$ & $<0.01$ & - & - & $1.46(1.38,1.54)$ & $<0.01$ \\
\hline$\geq 70$ & $1.18(1.11,1.26)$ & $<0.01$ & - & - & $1.18(1.11,1.26)$ & $<0.01$ \\
\hline \multicolumn{7}{|l|}{ Medical history } \\
\hline Hypertension & $1.13(1.07,1.20)$ & $<0.01$ & $1.04(0.98,1.10)$ & 0.21 & $1.02(0.96,1.08)$ & 0.58 \\
\hline Diabetes & $1.07(0.98,1.16)$ & 0.12 & $0.96(0.88,1.04)$ & 0.33 & $0.95(0.87,1.03)$ & 0.18 \\
\hline Hyperlipidemia & $1.22(1.12,1.34)$ & $<0.01$ & $1.10(1.01,1.20)$ & 0.04 & $1.09(1.00,1.20)$ & 0.06 \\
\hline Thyroid disease & $0.91(0.70,1.17)$ & 0.44 & $0.85(0.66,1.10)$ & 0.21 & - & - \\
\hline Anxiety & $1.13(0.76,1.69)$ & 0.54 & $0.98(0.65,1.46)$ & 0.90 & - & - \\
\hline Depression & $1.19(0.91,1.56)$ & 0.19 & $1.15(0.88,1.50)$ & 0.31 & $1.11(0.85,1.45)$ & 0.45 \\
\hline Heart disease & $1.38(1.22,1.56)$ & $<0.01$ & $1.20(1.07,1.36)$ & $<0.01$ & $1.17(1.04,1.33)$ & 0.01 \\
\hline Arthritis & $1.39(1.01,1.93)$ & 0.04 & $1.23(0.89,1.70)$ & 0.21 & $1.21(0.88,1.67)$ & 0.25 \\
\hline \multicolumn{7}{|c|}{ Sleep medicines } \\
\hline Estazolam & $1.59(1.43,1.77)$ & $<0.01$ & $1.44(1.29,1.60)$ & $<0.01$ & $1.42(1.27,1.59)$ & $<0.01$ \\
\hline Diazepam & $2.01(1.49,2.72)$ & $<0.01$ & $1.76(1.30,2.38)$ & $<0.01$ & $1.69(1.24,2.28)$ & $<0.01$ \\
\hline Alprazolam & $0.82(0.44,1.53)$ & 0.54 & $0.74(0.40,1.38)$ & 0.34 & - & - \\
\hline Lorazepam & $1.14(0.82,1.58)$ & 0.44 & $1.01(0.73,1.41)$ & 0.95 & - & - \\
\hline Trazodone & $0.83(0.35,1.99)$ & 0.68 & $0.82(0.34,1.97)$ & 0.66 & - & - \\
\hline Mirtazapine & $1.11(0.46,2.66)$ & 0.82 & $1.25(0.52,2.99)$ & 0.62 & - & - \\
\hline Doxepin & $2.31(0.33,16.33)$ & 0.40 & $1.85(0.26,13.08)$ & 0.54 & - & - \\
\hline Gabapentin & $1.75(1.41,2.17)$ & $<0.01$ & $1.51(1.22,1.88)$ & $<0.01$ & $1.51(1.22,1.88)$ & $<0.01$ \\
\hline Olanzapine & $0.78(0.53,1.14)$ & 0.20 & $0.75(0.51,1.10)$ & 0.14 & - & - \\
\hline
\end{tabular}

HR, Hazard ratio; Cl, Confidence interval.

Model 1: Univariate cox regression analysis, and no variables were adjusted.

Model 2: Sex and age adjusted.

Model 3: Variables with $P<0.20$ in univariate cox analysis were included for multivariate cox analysis.

The advantage of current study lies in the large sample size based on data from an integrated health care network covering a whole district in southeast China. Most of the previous studies used questionnaires $(19,20,26,27)$, which would cause recall bias. Our data from the medical information system can avoid such bias. The Cox proportional hazards model used in the current study is a reliable method to analyze the incidence of dry eye in sleep disorders patients, since it took the impact of time into consideration, which is different from the previous crosssectional population studies $(19,20,26,27)$. Besides, the paired cohort design using propensity score matching (1:1) increased the reliability of our study. However, limitations are unavoidable, and the main one is the diagnosis of DED and sleep disorder may not be in consistency among the hospitals and community health centers during the 8-year period. However, the paired cohort design made up for this drawback to a certain degree.
In conclusion, our findings suggest that sleep disorders is a potential risk factor for DED and the use of sleep medicines might be associated with dry eye development. Patients with sleep disorders or those using sleep medicines should be monitored for development of dry eye.

\section{DATA AVAILABILITY STATEMENT}

The raw data supporting the conclusions of this article will be made available by the authors, without undue reservation.

\section{ETHICS STATEMENT}

The studies involving human participants were reviewed and approved by Institutional Review Board of the affiliated Ningbo Eye Hospital of Wenzhou Medical University. Written 
informed consent for participation was not required for this study in accordance with the national legislation and the institutional requirements.

\section{AUTHOR CONTRIBUTIONS}

QZ designed the study. SL, FW, ZL, and KF analyzed the data. YS and JB checked the data. QZ and SL wrote the manuscript. HW revised the manuscript. PS, HL, and WC instructed the whole study and revised the manuscript. All authors contributed to the article and approved the submitted version.

\section{REFERENCES}

1. Kerkhof GA. Epidemiology of sleep and sleep disorders in The Netherlands. Sleep Med. (2017) 30:229-39. doi: 10.1016/j.sleep.2016.09.015

2. Govindarajan A, Urbach DR, Kumar M, Li Q, Murray BJ, Juurlink D, et al. Outcomes of daytime procedures performed by attending surgeons after night work. N Engl J Med. (2015) 373:845-53. doi: 10.1056/NEJMsa1415994

3. Altevogt BM CH. Sleep disorders and sleep deprivation: An unmet public health problem. J Am Acad Child Adolescent Psychiatry. (2008) 47:4734. doi: 10.1097/01.CHI.0000270812.55636.3b

4. Sabia S, Fayosse A, Dumurgier J, van Hees VT, Paquet C, Sommerlad A, et al. Association of sleep duration in middle and old age with incidence of dementia. Nat Commun. (2021) 12:2289. doi: 10.1038/s41467-021-22354-2

5. Shahrbabaki SS, Linz D, Hartmann S, Redline S, Baumert M. Sleep arousal burden is associated with long-term all-cause and cardiovascular mortality in 8001 community-dwelling older men and women. Eur Heart J. (2021) 42:2088-99. doi: 10.1093/eurheartj/ehab151

6. Vorona RD, Winn MP, Babineau TW, Eng BP, Feldman HR, Ware JC. Overweight and obese patients in a primary care population report less sleep than patients with a normal body mass index. Arch Intern Med. (2005) 165:25-30. doi: 10.1001/archinte.165.1.25

7. Meng L, Zheng Y, Hui R. The relationship of sleep duration and insomnia to risk of hypertension incidence: a meta-analysis of prospective cohort studies. Hypertens Res. (2013) 36:985-95. doi: 10.1038/hr.2013.70

8. Cappuccio FP, D’Elia L, Strazzullo P, Miller MA. Quantity and quality of sleep and incidence of type 2 diabetes: a systematic review and meta-analysis. Diabetes Care. (2010) 33:414-20. doi: 10.2337/dc09-1124

9. Aurora RN, Kim JS, Crainiceanu C, O’Hearn D, Punjabi NM. Habitual sleep duration and all-cause mortality in a general community sample. Sleep. (2016) 39:1903-9. doi: 10.5665/sleep.6212

10. Craig JP, Nichols KK, Akpek EK, Caffery B, Dua HS, Joo CK, et al. TFOS DEWS II definition and classification report. Ocul Surf. (2017) 15:27683. doi: 10.1016/j.jtos.2017.05.008

11. Stapleton F, Alves M, Bunya VY, Jalbert I, Lekhanont K, Malet F, et al. TFOS DEWS II epidemiology report. Ocul Surf. (2017) 15:33465. doi: 10.1016/j.jtos.2017.05.003

12. Kawashima M. Systemic health and dry eye. Invest Ophthalmol Vis Sci. (2018) 59:Des138-42. doi: 10.1167/iovs.17-23765

13. Vehof J, Sillevis Smitt-Kamminga N, Kozareva D, Nibourg SA, Hammond CJ. Clinical characteristics of dry eye patients with chronic pain syndromes. Am J Ophthalmol. (2016) 162:59-65.e2. doi: 10.1016/j.ajo.2015.11.017

14. Yu J, Asche CV, Fairchild CJ. The economic burden of dry eye disease in the United States: a decision tree analysis. Cornea. (2011) 30:37987. doi: 10.1097/ICO.0b013e3181f7f363

15. Wang MTM, Vidal-Rohr M, Muntz A, Diprose WK, Ormonde SE, Wolffsohn JS, et al. Systemic risk factors of dry eye disease subtypes: a New Zealand crosssectional study. Ocul Surf. (2020) 18:374-80. doi: 10.1016/j.jtos.2020.04.003

16. Vehof J, Snieder H, Jansonius N, Hammond CJ. Prevalence and risk factors of dry eye in 79,866 participants of the population-based Lifelines cohort study in the Netherlands. Ocul Surf. (2021) 19:83-93. doi: 10.1016/j.jtos.2020. 04.005

\section{FUNDING}

This work was financially supported by the Chinese National Key Research and Development Projects (2019YFC0840708).

\section{SUPPLEMENTARY MATERIAL}

The Supplementary Material for this article can be found online at: https://www.frontiersin.org/articles/10.3389/fmed. 2022.832851/full\#supplementary-material

17. Ayaki M, Tsubota K, Kawashima M, Kishimoto T, Mimura M, Negishi K. Sleep disorders are a prevalent and serious comorbidity in dry eye. Invest Ophthalmol Vis Sci. (2018) 59:Des143-50. doi: 10.1167/iovs.17-23467

18. Monjan AA. Perspective on sleep and aging. Front Neurol. (2010) 1:124. doi: 10.3389/fneur.2010.00124

19. Yu X, Guo H, Liu X, Wang G, Min Y, Chen SS, et al. Dry eye and sleep quality: a large community-based study in Hangzhou. Sleep. (2019) 42:zsz160. doi: 10.1093/sleep/zsz160

20. Lim EWL, Chee ML, Sabanayagam C, Majithia S, Tao Y, Wong TY, et al. Relationship between sleep and symptoms of tear dysfunction in singapore malays and Indians. Invest Ophthalmol Vis Sci. (2019) 60:188997. doi: 10.1167/iovs.19-26810

21. Wu M, Liu X, Han J, Shao T, Wang Y. Association between sleep quality, mood status, and ocular surface characteristics in patients with dry eye disease. Cornea. (2019) 38:311-7. doi: 10.1097/ICO.0000000000001854

22. Ayaki M, Kawashima M, Negishi K, Kishimoto T, Mimura M, Tsubota K. Sleep and mood disorders in women with dry eye disease. Sci Rep. (2016) 6:35276. doi: 10.1038/srep35276

23. Kawashima M, Uchino M, Yokoi N, Uchino Y, Dogru M, Komuro A, et al. The association of sleep quality with dry eye disease: the Osaka study. Clin Ophthalmol. (2016) 10:1015-21. doi: 10.2147/OPTH.S99620

24. Ayaki M, Kawashima M, Negishi K, Kishimoto T, Mimura M, Tsubota K. Sleep and mood disorders in dry eye disease and allied irritating ocular diseases. Sci Rep. (2016) 6:22480. doi: 10.1038/srep22480

25. Ayaki M, Kawashima M, Negishi K, Tsubota K. High prevalence of sleep and mood disorders in dry eye patients: survey of 1,000 eye clinic visitors. Neuropsychiatr Dis Treat. (2015) 11:889-94. doi: 10.2147/NDT.S81515

26. Hanyuda A, Sawada N, Uchino M, Kawashima M, Yuki K, Tsubota K, et al. Relationship between unhealthy sleep status and dry eye symptoms in a Japanese population: the JPHC-NEXT study. Ocul Surf. (2021) 21:30612. doi: 10.1016/j.jtos.2021.04.001

27. Magno MS, Utheim TP, Snieder H, Hammond CJ, Vehof J. The relationship between dry eye and sleep quality. Ocul Surf. (2021) 20:139. doi: 10.1016/j.jtos.2020.12.009

28. Kim KI, Park YS, Kim RH, Kim JH. Factors associated with dry eye symptoms in elderly Koreans: the fifth Korea National Health and nutrition examination survey 2010-2012. Korean J Fam Med. (2019) 40:2230. doi: 10.4082/kjfm.17.0058

29. Kawashima M, Yamada M, Shigeyasu C, Suwaki K, Uchino M, Hiratsuka Y, et al. Association of systemic comorbidities with dry eye disease. J Clin Med. (2020) 9:2040. doi: 10.3390/jcm9072040

30. Koçer E, Koçer A, Özsütçü M, Dursun AE, Krpnar I. Dry eye related to commonly used new antidepressants. J Clin Psychopharmacol. (2015) 35:4113. doi: 10.1097/JCP.0000000000000356

31. Zhang S, Hong J. Risk factors for dry eye in Mainland China: a multicenter cross-sectional hospital-based study. Ophthalmic Epidemiol. (2019) 26:393-9. doi: 10.1080/09286586.2019.1632905

32. Wang JB, Huang QC, Hu SC, Zheng PW, Shen P, Li D, et al. Baseline and longitudinal change in blood pressure and mortality in a Chinese cohort. J Epidemiol Community Health. (2018) 72:108390. doi: 10.1136/jech-2018-211050 
33. Aurora RN, Zak RS, Auerbach SH, Casey KR, Chowdhuri S, Karippot A, et al. Best practice guide for the treatment of nightmare disorder in adults. J Clin Sleep Med. (2010) 6:389-401. doi: 10.5664/jcsm.27883

34. Okun ML, Ebert R, Saini B. A review of sleep-promoting medications used in pregnancy. Am J Obstet Gynecol. (2015) 212:428-41. doi: 10.1016/j.ajog.2014.10.1106

35. Lee W, Lim SS, Won JU, Roh J, Lee JH, Seok H, et al. The association between sleep duration and dry eye syndrome among Korean adults. Sleep Med. (2015) 16:1327-31. doi: 10.1016/j.sleep.2015.06.021

36. Han KT, Nam JH, Park EC. Do sleep disorders positively correlate with dry eye syndrome? Results of national claim data. Int J Environ Res Public Health. (2019) 16:878. doi: 10.3390/ijerph16050878

37. Li S, Ning K, Zhou J, Guo Y, Zhang H, Zhu Y, et al. Sleep deprivation disrupts the lacrimal system and induces dry eye disease. Exp Mol Med. (2018) 50:e451. doi: 10.1038/emm.2017.285

38. Palma JA. Autonomic dysfunction in sleep disorders: introduction to the series. Clin Auton Res. (2018) 28:507-8. doi: 10.1007/s10286-018-0571-6

39. Tang L, Wang X, Wu J, Li SM, Zhang Z, Wu S, et al. Sleep deprivation induces dry eye through inhibition of PPAR $\alpha$ expression in corneal epithelium. Invest Ophthalmol Vis Sci. (2018) 59:5494-508. doi: 10.1167/iovs.18-24504

40. Dartt DA. Regulation of tear secretion. Adv Exp Med Biol. (1994) 350:19. doi: 10.1007/978-1-4615-2417-5_1

41. Dartt DA. Neural regulation of lacrimal gland secretory processes: relevance in dry eye diseases. Prog Retin Eye Res. (2009) 28:15577. doi: 10.1016/j.preteyeres.2009.04.003
42. Sleep disorders group soN, Chinese Medical Association. Guidelines for the diagnosis and treatment of insomnia in Chinese adults. Chin J Neurol. (2018) 51:324-35. doi: 10.21037/jtd.2019.06.09

43. Richa S, Yazbek JC. Ocular adverse effects of common psychotropic agents: a review. CNS Drugs. (2010) 24:50126. doi: 10.2165/11533180-000000000-00000

Conflict of Interest: The authors declare that the research was conducted in the absence of any commercial or financial relationships that could be construed as a potential conflict of interest.

Publisher's Note: All claims expressed in this article are solely those of the authors and do not necessarily represent those of their affiliated organizations, or those of the publisher, the editors and the reviewers. Any product that may be evaluated in this article, or claim that may be made by its manufacturer, is not guaranteed or endorsed by the publisher.

Copyright (C) 2022 Zheng, Li, Wen, Lin, Feng, Sun, Bao, Weng, Shen, Lin and Chen. This is an open-access article distributed under the terms of the Creative Commons Attribution License (CC BY). The use, distribution or reproduction in other forums is permitted, provided the original author(s) and the copyright owner(s) are credited and that the original publication in this journal is cited, in accordance with accepted academic practice. No use, distribution or reproduction is permitted which does not comply with these terms. 